\title{
Ressecção primária incompleta do câncer de pele não melanoma em um hospital universitário
}

\author{
Incomplete primary resection of non-melanoma skin cancer in a university hospital
}

Resección primaria incompleta de cáncer de piel no melanoma en un hospital universitario

Maria de Fátima de Brito Coutinho Nogueira Lima ${ }^{1}$, Ryan Nogueira Lima², Luis Gustavo Cavalcante Reinaldo ${ }^{3}$, Auriane de Sousa Alencar ${ }^{4 *}$, Carlos Alberto Rosado da Silva Filho ${ }^{3}$, Tarso Buaiz Pereira Martins ${ }^{3}$, Virgínia Portela Cardoso ${ }^{3}$, Anatália de Oliveira Viana ${ }^{5}$, Julciele Cícera da Silva $^{3}$, Mauri Brandão de Medeiros Júnior ${ }^{3}$.

\section{RESUMO}

Objetivo: Avaliar a ressecção primária incompleta do câncer de pele não melanoma em um serviço de cirurgia plástica de um hospital universitário. Métodos: Delineamento transversal analítico, com coleta de dados retrospectivos, realizado em um hospital universitário do nordeste do Brasil, em janeiro de 2021. A população do estudo compreendeu pacientes submetidos a cirurgia para exérese de lesões sugestivas de câncer de pele no serviço de Cirurgia Plástica, no período de março 2019 a março de 2020. Uma amostra de conveniência composta por 104 participantes foi avaliada. Foram coletadas variáveis demográficas e epidemiológicas relacionadas às lesões. Foram calculadas estatísticas uni e bivariadas. Resultados: Os pacientes apresentaram média de idade próxima aos 70 anos, sendo que $23,1 \%$ apresentaram lesões múltiplas. A prevalência de ressecção incompleta foi de $29,8 \%$. Maior número de lesões por paciente $(p=0,009)$, lesões com diâmetro superior a $2 \mathrm{~cm} \quad(p=0,008)$, localizadas na face $(p=0,048)$, além de carcinomas basocelulares dos subtitpos esclerodermiforme $(p=0,042)$ e infiltrativo $(p=0,026)$ foram significativamente relacionados à ressecção primária incompleta do câncer de pele não melanoma. Conclusão: A prevalência de ressecção primária incompleta do câncer de pele não melanoma foi alta e foi relacionada às características das lesões.

Palavras-chave: Câncer de pele, Carcinoma basocelular, Margens cirúrgicas.

\begin{abstract}
Objective: To evaluate the incomplete primary resection of non-melanoma skin cancer in a plastic surgery service at a university hospital. Methods: Analytical cross-sectional design, with retrospective data collection, carried out in a university hospital in northeastern Brazil, in January 2021. The study population comprised patients undergoing surgery for excision of lesions suggestive of skin cancer in a Plastic Surgery service from March 2019 to March 2020. A convenience sample of 104 participants was evaluated. Demographic and epidemiological variables were collected. Univariate and bivariate statistics were calculated. Results: The patients had an average age close to 70 years, with $23.1 \%$ having multiple injuries. The prevalence of incomplete resection was $29.8 \%$. Higher number of lesions per patient $(p=0.009)$, lesions with a diameter greater than $2 \mathrm{~cm}(p=0.008)$, located on the face $(p=0.048)$, in addition to sclerodermiform $(p=0.042)$ and infiltrative $(p=0.026)$ subtypes of basal cell carcinomas were significantly related to incomplete primary resection of non-melanoma skin cancer. Conclusion: The prevalence of incomplete primary resection of non-melanoma skin cancer was high and was related to lesion characteristics.
\end{abstract}

Keywords: Skin cancer, Carcinoma basal cell, Surgical margins.

\footnotetext{
${ }^{1}$ Hospital Walter Cantídio, Fortaleza - CE.

${ }^{2}$ Hospital de Messejana Dr. Carlos Alberto Stuart Gomes, Fortaleza - CE.

${ }^{3}$ Hospital Universitário da Universitário da Universidade Federal do Piauí, Teresina - PI.

${ }^{4}$ Hospital Infantil Lucídio Portella, Teresina - PI. *E-mail: aurialencar@hotmail.com

5 Universidade Federal do Piauí, Teresina - PI.
} 


\section{RESUMEN}

Objetivo: Evaluar la resección primaria incompleta de un cáncer de piel no melanoma en un servicio de cirugía plástica de un hospital universitario. Métodos: Diseño analítico transversal, con recolección de datos retrospectiva, realizado en un hospital universitario del noreste de Brasil, en enero de 2021. La población de estudio estuvo constituida por pacientes intervenidos de escisión de lesiones sugestivas de cáncer de piel en el servicio de Cirugía Plástica, de marzo de 2019 a marzo de 2020. Se evaluó una muestra de conveniencia de 104 participantes. Se recogieron variables demográficas y epidemiológicas relacionadas con las lesiones. Se calcularon estadísticas univariadas y bivariadas. Resultados: Los pacientes tenían una edad media cercana a los 70 años, con un $23,1 \%$ de lesiones múltiples. La prevalencia de resección incompleta fue del $29,8 \%$. Mayor número de lesiones por paciente $(p=0,009)$, lesiones con diámetro mayor a $2 \mathrm{~cm}(p=0,008)$, localizadas en la cara $(p=0,048)$, además de carcinomas basocelulares del esclerodermiforme $(p=0,042)$ e infiltrante $(p=0.042)=0.026)$ se relacionaron significativamente con la resección primaria incompleta del cáncer de piel no melanoma. Conclusión: La prevalencia de resección primaria incompleta del cáncer de piel no melanoma fue alta y las características de las lesiones estaban relacionadas.

Palabras clave: Cáncer de piel, Carcinoma basocelular, Márgenes quirúrgicos positivos.

\section{INTRODUÇÃO}

A neoplasia maligna de pele é o câncer mais prevalente no Brasil tendo origem multifatorial e tem influência da exposição prolongada aos raios ultravioletas (GAMONAL AC, et al., 2020; HUANG T, et al., 2019) A principal modalidade de tratamento dessa patologia é a exérese cirúrgica das lesões. A ressecção oncológica completa dos carcinomas de pele na primeira abordagem reflete positivamente no prognóstico desses pacientes, diminuindo, sobretudo, a morbidade decorrente de alterações funcionais e estéticas desencadeadas pela invasão tumoral e, ainda, por reabordagens para exérese completa do câncer (MELO R, et al., 2020; BADASH I, et al., 2019; LAI V, et al., 2018).

Os carcinomas de pele são considerados tumores de alto grau de malignidade, com intensa capacidade de invasão e destruição tecidual, embora com limitado poder de metastização. Diversos fatores têm sido associados à ocorrência de câncer de pele não melanoma, em especial a faixa etária mais avançada, cor da pele clara e sexo masculino (DUARTE F, et al., 2018; SILVA R e DIAS M, 2017).

Além disso, os cânceres de pele dividem-se em câncer de pele não melanoma e câncer de pele melanoma. Os tipos histológicos mais comuns do câncer de pele não melanoma são carcinoma basocelular (CBC) e carcinoma espinocelular (CEC), sendo o primeiro mais comum. Já o segundo origina-se de queratinócitos epidérmicos ou estruturas anexiais e, embora seja raro, os carcinomas de células escamosas podem migrar para gânglios linfáticos regionais, assim como para outros locais, como osso, cérebro e pulmões (KALLINI JR, et al., 2015).

Os CBC são classificados em diversos subtipos, com comportamento mais ou menos agressivo. Embora, em regra, tenham crescimento lento e se apresentem como lesões únicas, não raras são a ocorrência de várias lesões simultâneas ou subsequentes. Na abordagem terapêutica do $\mathrm{CBC}$, a cirurgia convencional (cirurgia excecional) é a técnica dominante, em que pese existirem diversas outras modalidades de terapia (DOMÍNGUEZ C e CELIS J, 2018)

O tratamento cirúrgico de um CBC é realizado com intenção curativa. Para tanto, um fator importante é a avaliação da margem pós-operatória, com a definição da borda clínica do tumor. É preciso selecionar a margem buscando uma excisão completa sem sacrificar o excesso de tecido normal. A identificação precisa da borda do tumor requer um exame cuidadoso com boa luz, ampliação da lupa e palpação. À borda do tumor clínico, uma margem é adicionada para explicar qualquer extensão subclínica antes da excisão (FAHRADYAN A, et al., 2017).

A taxa de excisão incompleta varia com a margem cirúrgica, localização do tumor, tamanho, subtipo histológico, tratamento anterior, fatores do cirurgião e do paciente. O tamanho da lesão maior que $2 \mathrm{~cm}$ de 
diâmetro é um dos principais fatores de risco para a recorrência e metastização da neoplasia. Também são frequentemente associados a margem incompleta a localização na parte central da face e orelhas, lesões com longo tempo de aparecimento, tipo histológico agressivo e (NIGHTINGALE J, et al., 2020; COLLINS A, et al., 2019; NAPOLI J, et al., 2021).

Os dados deste estudo podem contribuir com o aprimoramento da assistência em saúde da população acometida por câncer de pele não melanoma, a partir da identificação de fatores relacionados à exérese oncológica incompleta das lesões. A exérese cirúrgica é a principal modalidade de tratamento para o câncer de pele, diante disso, entender quais fatores relacionam-se a ressecções incompletas possibilitará o desenvolvimento no tratamento dessa patologia, que corresponde ao câncer mais prevalente em nossa população (SILVA P, et al., 2021).

Este estudo objetivou avaliar a ressecção primária incompleta do câncer de pele não melanoma em um serviço de cirurgia plástica de um hospital universitário do nordeste.

\section{MÉTODOS}

Delineamento transversal analítico, com coleta de dados retrospectivos, realizado em um hospital universitário do Nordeste, que compreende uma referência regional para o diagnóstico e tratamento de pacientes com câncer. O estudo foi desenvolvido em janeiro de 2021.

A população do estudo compreendeu pacientes submetidos a cirurgia para exérese de lesões sugestivas de câncer de pele no serviço de Cirurgia Plástica, no período de março 2019 a março de 2020, que totalizaram 153 pacientes. Para composição da amostra foram critérios de inclusão: ter idade igual ou superior a 18 anos e ter realizado ressecção cirúrgica de câncer de pele não melanoma.

Foram critérios de exclusão: não comparecer para seguimento pós-operatório após exérese excisional da lesão, apresentar câncer de pele recidivado ou não ser localizado para avaliação do procedimento cirúrgico realizado. A amostragem foi não probabilística, do tipo por conveniência, e buscou incluir todos os pacientes que atendiam aos critérios de elegibilidade para o estudo no período determinado. Ao final, a amostra ficou composta por 104 participantes.

Foi utilizado um formulário de coleta de dados, contendo variáveis demográficas (idade, sexo e cor/raça autodeclarada) e epidemiológicas (quantidade e tamanho das lesões, localização anatômica e tipo histológico) relacionadas às lesões. A coleta de dados foi realizada em fontes de informações intrahospitalares para identificação dos pacientes que atendiam aos critérios do estudo.

Após a etapa de identificação da população, foi realizada a análise individual dos prontuários para elaboração da lista de pacientes que atendiam aos critérios de elegibilidade para o estudo. Em seguida, foi realizado o contato para explicação dos objetivos do estudo e obtenção do consentimento para participação no estudo.

A exérese cirúrgica dos cânceres de pele não seguiu a técnica micrográfica de Mohs e foi realizada reconstrução de forma heterogênea. Foi realizada a ressecção com 3 a $5 \mathrm{~mm}$ de margem, e classificado o tipo histológico em: nodular ou sólido, micronodular, esclerodermiforme, adenoide, ulcerado, superficial ou pigmentado. A classificação de Broders foi empregada para os carcinomas espinocelulares. Levando em consideração a porcentagem de células indiferenciadas, onde grau I bem diferenciado, grau II, moderadamente diferenciado, grau III, mal diferenciado.

Os dados foram processados no software IBM ${ }^{\circledR}$ SPSS $\AA$, versão 26.0. Foram calculadas estatísticas univaridas, como média e desvio padrão, para as variáveis quantitativas, e frequências para as qualitativas. $\mathrm{Na}$ análise inferencial, a variável dependente foi definida como a classificação da resseção (incompleta versus completa).

Para as variáveis independentes quantitativas, a normalidade dos dados foi verificada por meio do teste de Kolmogorov-Smirnov e foi utilizado o teste t de Student. Para as independentes qualitativas, foi uitlizado o teste Qui-Quadrado de Pearson e, quando não atendidos os pressupostos desse teste, foi realizado o 
teste Exato de Fisher. Para as associações significativas, foi calculada a Razão de Prevalência (RP) e seu respectivo Intervalo de Confiança (IC95\%). Os percentuais das tabelas de contingência foram calculados com base no total da amostra. As relações foram consideradas significativas quando $p<0,05$.

Foram seguidas as normatizações éticas nacionais e internacionais para pesquisas com seres humanos. O estudo foi autorizado pelo hospital universitário e foi aprovado por Comitê de Ética em Pesquisa (CEP), sob parecer n. $.4 .447 .114 / 2021$. Todos os participantes assinaram o Termo de Consentimento Livre e Esclarecido (TCLE), em duas vias, e foi apresentado o termo de compromisso de utilização de dados.

\section{RESULTADOS}

Dentre os 104 pacientes submetidos à ressecção primária do câncer de pele não melanoma no hospital universitário no período de março de 2019 a março de 2020 que compuseram a amostra do estudo, 24 $(23,1 \%)$ apresentaram duas ou mais lesões, totalizando 146 lesões. A amostra foi caracterizada quanto aos aspectos demográficos e clínicos (Tabela 1). Foram avaliados o tamanho das lesões (Tabela 2), a localização anatômica (Tabela 3) e os tipos de lesões (Tabela 4) dos pacientes.

Tabela 1 - Características demográficas e clínicas dos pacientes que realizaram ressecção primária do câncer de pele não melanoma $(n=104)$.

\begin{tabular}{|c|c|c|c|c|}
\hline Característica & Incompleta & Completa & Total & $p$ \\
\hline Idade* & $68,8 \pm 13,9$ & $70,4 \pm 13,3$ & $69,9 \pm 13,4$ & $0,593^{t}$ \\
\hline Sexo & & & & $0,868^{9}$ \\
\hline Masculino & $12(11,5 \%)$ & $27(26,0 \%)$ & $39(37,5 \%)$ & \\
\hline Feminino & $19(18,3 \%)$ & $46(44,2 \%)$ & $65(62,5 \%)$ & \\
\hline Cor/raça autodeclarada & & & & - \\
\hline Parda & $30(28,8 \%)$ & $61(58,7 \%)$ & $91(87,5 \%)$ & \\
\hline Branca & $1(1,0 \%)$ & $9(8,6 \%)$ & $10(9,6 \%)$ & \\
\hline Preta & - & $2(1,9 \%)$ & $2(1,9 \%)$ & \\
\hline Amarela & - & $1(1,0 \%)$ & $1(1,0 \%)$ & \\
\hline Número total de lesões/paciente & $1,9 \pm 1,3$ & $1,2 \pm 0,6$ & & $0,009^{t}$ \\
\hline Número de lesões $\leq 0,5 \mathrm{~cm}$ & $0,5 \pm 0,9$ & $0,3 \pm 0,5$ & & $0,177^{t}$ \\
\hline Número de lesões $>0,5$ e $\leq 1,0 \mathrm{~cm}$ & $0,4 \pm 0,6$ & $0,4 \pm 0,6$ & & $0,899^{t}$ \\
\hline Número de lesões $>1,0$ e $\leq 2,0 \mathrm{~cm}$ & $0,2 \pm 0,4$ & $0,2 \pm 0,5$ & & $0,679^{t}$ \\
\hline Número de lesões $>2,0 \mathrm{~cm}$ & $0,7 \pm 0,9$ & $0,3 \pm 0,5$ & & $0,015^{t}$ \\
\hline Total & $31(29,8 \%)$ & $73(70,2 \%)$ & $104(100,0 \%)$ & \\
\hline
\end{tabular}

Legenda: *: média \pm desvio padrão; $p$ : significância do teste; $t$ : teste $\mathrm{t}$ de Student; $q$ : qui-quadrado de Pearson. Fonte: Brito MFC, et al., 2021.

$\mathrm{Na}$ totalidade dos pacientes estudados, a média ( \pm desvio padrão) de idade, de forma geral, foi de 69,9 $( \pm 13,4)$ anos, sendo que a idade média dos pacientes com margem cirúrgica comprometida foi de 68,8 $( \pm 13,9)$ anos. Predominaram pacientes do sexo feminino $65(62,5 \%)$, porém, a prevalência de ressecções com margem cirúrgica comprometida no sexo masculino foi maior, atingindo $12(11,5 \%)$ dentre os 39 $(37,5 \%)$ pacientes avaliados. Quanto à cor da pele, 91 (87,5\%) autodeclararam-se pardos e, dentre eles, 30 $(28,8 \%)$ apresentaram margem cirúrgica comprometida. Verificou-se que pacientes que apresentaram ressecção incompleta de pelo menos uma lesão tinha número total de lesões significativamente superior em relação aos com ressecção completa $(p=0,009), 1,9( \pm 1,3)$ versus $1,2( \pm 0,6)$, especialmente lesões de tamanho superior a $2 \mathrm{~cm}$, cujo número médio foi significativamente maior dentre os com ressecção incompleta $(p=0,015), 0,7( \pm 0,9)$ versus $0,3( \pm 0,5)$, conforme a Tabela 1. 
Tabela 2 - Distribuições das lesões dos pacientes por tamanho e classificação da ressecção primária do câncer de pele não melanoma $(n=146)$.

\begin{tabular}{|c|c|c|c|c|}
\hline Tamanho da lesão & Incompleta & Completa & Total & $p$ \\
\hline Lesão com tamanho $\leq 0,5 \mathrm{~cm}$ & & & & 0,698 \\
\hline$\overline{\operatorname{Sim}}$ & $10(6,8 \%)$ & $32(21,9 \%)$ & $42(28,8 \%)$ & \\
\hline Não & $28(19,2 \%)$ & $76(52,1 \%)$ & $104(71,2 \%)$ & \\
\hline Lesão com tamanho $>0,5 \mathrm{a} \leq 1,0 \mathrm{~cm}$ & & & & 0,140 \\
\hline$\overline{\operatorname{Sim}}$ & $6(4,1 \%)$ & $30(20,5 \%)$ & $36(24,7 \%)$ & \\
\hline Não & $32(21,9 \%)$ & $78(53,4 \%)$ & $110(75,3 \%)$ & \\
\hline Lesão com tamanho $>1,0$ a $\leq 2,0 \mathrm{~cm}$ & & & & 0,325 \\
\hline$\overline{\operatorname{Sim}}$ & $5(3,4 \%)$ & $22(15,1 \%)$ & $27(18,5 \%)$ & \\
\hline Não & $33(22,6 \%)$ & $86(58,9 \%)$ & $119(81,5 \%)$ & \\
\hline Lesão com tamanho $>2,0 \mathrm{~cm}$ & & & & 0,008 \\
\hline$\overline{\operatorname{Sim}}$ & $17(11,6 \%)$ & $24(16,4 \%)$ & $41(28,1 \%)$ & \\
\hline Não & $21(14,4 \%)$ & $84(57,5 \%)$ & $105(71,9 \%)$ & \\
\hline Total & $38(26,0 \%)$ & $108(74,0 \%)$ & $146(100,0 \%)$ & \\
\hline
\end{tabular}

Legenda: p: significância do teste Qui-Quadrado de Pearson. Fonte: Brito MFC, et al., 2021.

Quanto ao tamanho das lesões em seu maior diâmetro, $42(28,8 \%)$ eram menor ou igual a $0,5 \mathrm{~cm}$, dentre as quais $10(6,8 \%)$ foram ressecadas de forma incompleta. Foram avaliadas $36(24,7 \%)$ lesões maiores que $0,5 \mathrm{~cm}$ e menores ou iguais a $1 \mathrm{~cm}$ e $6(4,1 \%)$ apresentaram margem cirúrgica comprometida. Dentre as $27(18,5 \%)$ com tamanho superior a $1 \mathrm{~cm}$ até $2 \mathrm{~cm}$, apenas $5(3,4 \%)$ foram ressecadas com margem cirúrgica comprometida. Dentre as $41(28,1 \%)$ lesões de tamanho superior a $2 \mathrm{~cm}, 17(11,6 \%)$ foram ressecadas de forma incompleta, com $\mathrm{RP}=2,073$ (IC95\%=1,223-3,515), de modo que lesões com esse tamanho em seu maior diâmetro apresentaram prevalência aproximadamente 2,1 vezes maior de comprometimento de margens ( $p=0,008)$, conforme apresentado na Tabela 2.

Tabela 3 - Localização anatômica das lesões dos pacientes conforme classificação da ressecção primária do câncer de pele não melanoma $(n=146)$.

\begin{tabular}{lcccc}
\hline \multicolumn{1}{c}{ Localização anatômica } & Incompleta & Completa & Total & $\boldsymbol{p}$ \\
\hline Face & & & $\mathbf{0 , 0 4 8 ^ { q }}$ \\
\hline Sim & $34(23,3 \%)$ & $80(54,8 \%)$ & $114(78,1 \%)$ & \\
Não & $4(2,7 \%)$ & $28(19,2 \%)$ & $32(21,9 \%)$ & \\
\hline Frontal $^{*}$ & $5(4,4 \%) 41.8$ & $7(6,1 \%)$ & $12(10,5 \%)$ & $0,338^{f}$ \\
\hline Orelha $^{*}$ & $2(1,8 \%) 40.9$ & $3(2,6 \%)$ & $5(4,4 \%)$ & $0,634^{f}$ \\
\hline Região periauricular $^{*}$ & $4(3,5 \%) 33.4$ & $8(7,0 \%)$ & $12(10,5 \%)$ & $0,749^{f}$ \\
\hline Pálpebras $^{*}$ & $6(5,3 \%) 46.5$ & $7(6,1 \%)$ & $13(11,4 \%)$ & $0,203^{f}$ \\
\hline Região malar $^{*}$ & $5(4,4 \%) 20.8$ & $19(16,7 \%)$ & $24(21,1 \%)$ & $0,279^{q}$ \\
\hline Lábio* $^{*}$ & - & $3(2,6 \%)$ & $3(2,6 \%)$ & $0,553^{f}$ \\
\hline Região perioral $^{*}$ & - & $11(9,6 \%)$ & $11(9,6 \%)$ & $\mathbf{0 , 0 3 2 ^ { f }}$ \\
\hline Nariz* & & & $34(29,8 \%)$ & $0,405^{q}$ \\
\hline Tronco & $12(10,5 \%) 35.2$ & & $0,159^{q}$ \\
\hline Sim & $22(19,3 \%)$ & $19(13 \%)$ & \\
Não & $2(1,4 \%)$ & $17(11,6 \%)$ & $127(87 \%)$ & \\
Membros & $36(24,7 \%)$ & $91(62,3 \%)$ & & $1,000^{q}$ \\
\hline Sim & $2(1,4 \%)$ & $7(4,8 \%)$ & $9(6,2 \%)$ & \\
Não & $36(24,7 \%)$ & $101(69,2 \%)$ & $137(93,8 \%)$ & \\
\hline Total & $\mathbf{3 8 ( 2 6 , 0 \% )}$ & $\mathbf{1 0 8 ( 7 4 , 0 \% )}$ & $\mathbf{1 4 6 ( 1 0 0 , 0 \% )}$ & \\
\hline
\end{tabular}

Legenda: *: frequências apresentadas apenas para a categoria "sim"; p: significância do teste; q: quiquadrado de Pearson; f: teste exato de Fisher. Fonte: Brito, M. F. C., et al., 2021. 
Lesões com ressecção incompleta apresentaram frequência significativamente maior na face $34(23,3 \%)$, com $\mathrm{RP}=2,386$ (IC95\%=1,915-6,225), correspondendo a uma prevalência 2,4 vezes maior de comprometimento de margens em lesões localizadas na face $(p=0,048)$. A localização anatômica mais prevalente na face, dentre as $114(78,1 \%)$ lesões avaliadas, foi o nariz $34(29,8 \%)$, seguido da região malar $24(21,1 \%)$. Na região perioral, foi significativa a frequência de lesões com ressecção completa $11(9,6 \%)$, com $\mathrm{RP}=1,493$ (IC95\%=1,304-1,709), de modo que lesões nessa região apresentaram uma prevalência $49,3 \%$ maior de margens livres. As lesões em tronco e em membros apresentaram maior frequência de ressecção completa, em 17 (11,6\%) e 7 (4,8\%) pacientes, respectivamente, conforme Tabela 3.

Tabela 4 - Tipos das lesões dos pacientes conforme classificação da ressecção primária do câncer de pele não melanoma ( $n=146)$. Teresina, PI, Brasil, 2021

\begin{tabular}{|c|c|c|c|c|}
\hline Característica & Incompleta & Completa & Total & $p$ \\
\hline Carcinoma basocelular & & & & $0,325^{9}$ \\
\hline Sim & $31(21,2)$ & $95(65,1)$ & $126(86,3)$ & \\
\hline Não & $7(4,8)$ & $13(8,9)$ & $20(13,7)$ & \\
\hline Nodular/sólido* & $13(10,3)$ & $58(46,0)$ & $71(56,3)$ & $0,062^{q}$ \\
\hline Micronodular* & $3(2,3)$ & $5(4,0)$ & $8(6,3)$ & $0,405^{f}$ \\
\hline Esclerodermiforme* & $7(5,6)$ & $7(5,6)$ & $14(11,2)$ & $0,042^{f}$ \\
\hline Adenoide* $^{*}$ & $1(0,8)$ & $12(9,5)$ & $13(10,3)$ & $0,184^{f}$ \\
\hline Ulcerado* $^{*}$ & $16(12,7)$ & $39(31,0)$ & $55(43,7)$ & $0,303^{q}$ \\
\hline Superficial ${ }^{*}$ & $2(1,6)$ & $8(6,3)$ & $10(7,9)$ & $1,000^{f}$ \\
\hline Pigmentado* & $5(4,0)$ & $14(11,1)$ & $19(15,1)$ & $1,000^{f}$ \\
\hline Infiltrativo* & $10(7,9)$ & $12(9,5)$ & $22(17,5)$ & $0,026^{f}$ \\
\hline Carcinoma espinocelular & & & & $0,325^{9}$ \\
\hline Sim & $7(4,8)$ & $13(8,9)$ & $20(13,7)$ & \\
\hline Não & $31(21,2)$ & $95(65,1)$ & $126(86,3)$ & \\
\hline Lesões de grau I* & $3(15,0)$ & $2(10,0)$ & $5(25,0)$ & $0,290^{f}$ \\
\hline Lesões de grau II* & $2(10,0)$ & - & $2(10,0)$ & $0,111^{f}$ \\
\hline Lesões de grau III* & - & $1(5,0)$ & $1(5,0)$ & $1,000^{f}$ \\
\hline In situ & $2(10,0)$ & $10(50,0)$ & $12(60,0)$ & \\
\hline Total & $38(26,0 \%)$ & $108(74,0 \%)$ & $146(100,0 \%)$ & \\
\hline
\end{tabular}

Legenda: *: frequências apresentadas apenas para a categoria "sim"; p: significância do teste; q: quiquadrado de Pearson; $f$ : teste exato de Fisher

Fonte: Brito MFC, et al., 2021.

Quanto aos tipos histológicos das lesões, predominou o adenocarcinoma basocelular 126 (86,3\%). No grupo de pacientes com ressecção incompleta e carcinoma basocelular $31(21,2 \%)$, foi significativamente prevalente o padrão esclerodermiforme, com $\mathrm{RP}=2,333$ (IC95\%=1,240-4,392), em que a presença desse padrão representou uma prevalência 2,3 vezes maior de comprometimento de margens de lesões de câncer de pele não melanoma $(p=0,042)$. Outro padrão com presença significativa de ressecção incompleta foi o infiltrativo, com $\mathrm{RP}=2,251$ (IC95\%=1,240-4,086), em que pacientes com carcinoma basocelular com esse padrão apresentaram prevalência 2,2 vezes maior de ressecção incompleta $(p=0,026)$. Dentro do grupo CEC, o subtipo de Broders mais prevalente foi o grau I, compreendendo 5 (25,0\%) lesões, sendo identificados ainda $12(60,0)$ Carcinomas epidermoides in situ, conforme a Tabela 4.

\section{DISCUSSÃO}

A prevalência de ressecção primária incompleta do câncer de pele não melanoma no hospital universitário em foco foi de $29,8 \%$. Não existe consenso na literatura para a classificação do índice de 
margem cirúrgica comprometida nas exéreses para tratamento do câncer de pele. Estudo transversal retrospectivo realizado na Colômbia, com 145 pacientes com diagnóstico de câncer de pele submetidos a tratamento cirúrgico, encontrou uma prevalência de $26,5 \%$ de ressecções incompletas (BILDEN TT, et al., 2019).

A média de idade dos participantes do presente estudo, de forma geral, foi próxima aos 70 anos, o que corrobora com dados apontados na literatura, em que essa característica está diretamente correlacionada à incidência de câncer de pele. Verificou-se, ainda, uma prevalência importante de lesões múltiplas na amostra $(23,1 \%)$ e um número significativamente maior de lesões no grupo com ressecção incompleta. Estudo transversal desenvolvido no serviço de cirurgia plástica de um hospital de São Paulo, Brasil, avaliou prontuários médicos de 145 pacientes que foram tratados de câncer de pele não melanoma e encontrou média de idade 69,5 anos, sendo que 15,9\% exibiram lesões múltiplas, resultando em um total de 168 tumores. O CBC foi o mais comum (87,6\%), do tipo sólido ou nodular (44,1\%), enquanto o CEC de Broders I representou 42,1\% dos casos epidermoides (BROETTO J, et al., 2012).

Embora o pico de incidência de CBC ocorra por volta dos 40 anos de idade, sua incidência continua a aumentar, dobrando a cada 25 anos. O carcinoma de células basais é raro em crianças e aumenta em frequência com a idade, possivelmente como resultado do aumento da exposição ao sol, com uma idade mediana no diagnóstico de 68 anos ${ }^{(14)}$. Ademais, aponta-se um período de latência de 20 a 50 anos entre o tempo de exposição aos raios ultravioleta e o aparecimento do tumor. Em crianças e adultos jovens, o CBC está associado a síndromes genéticas, como síndrome de Bazex, síndrome do nevo basocelular (também chamada de síndrome de Gorlin) e xeroderma pigmentoso (QUAZI SJ, et al., 2020).

O sexo feminino predominou na população estuda correspondendo a $62,5 \%$, porém, proporcionalmente, houve menos ressecção com margem cirúrgica comprometida nesse grupo quando comparado ao sexo masculino. A distribuição por sexo dos cânceres de pele não melanoma não tem padrão definido na literatura, variando amplamente de país para país, inclusive sobre qual sexo prevalece. A proporção relatada de homem para mulher daqueles afetados com CBC varia de 1,3:1 a 1,6:1 (NAHHAS AF, et al., 2017).

Uma revisão retrospectiva de registros de 13.457 CBC em 10.245 pacientes em um centro de dermatopatologia na França encontrou a proporção de homem para mulher de 0,92:1(16). O desenvolvimento de CBC avançado ocorre principalmente em homens (CIVES M, et al., 2017). Outro estudo, realizado na Nova Zelândia, cujo desfecho primário foi taxa de margem positiva e próxima $(\leq 1 \mathrm{~mm})$ após excisão cirúrgica e fatores preditores, verificou uma probabilidade duas vezes maior de margens próximas em mulheres.

Neste estudo, não foi verificada associação entre cor/raça e ressecção incompleta de lesões do câncer de pele não melanoma, com alta prevalência de indivíduos autodeclarados pardos. O câncer de pele desenvolve-se com mais frequência em indivíduos caucasianos expostos a fatores ambientais, além de radiação ultravioleta, tabagismo, infecções crônicas e imunossupressores, ou com histórico genético peculiar (CIVES M, et al., 2017). A baixa frequência de pacientes brancos pode estar relacionada a aspectos culturais regionais, devido à forte miscigenação, assim como há possibilidade de viéses de registros nos prontuários médicos.

Pacientes com lesões de tamanho superior a $2 \mathrm{~cm}$ tiveram o dobro da prevalência de ressecção incompleta na amostra do estudo. O tamanho das lesões influencia diretamente no risco de ressecção incompleta destas. As áreas acometidas por carcinomas de pele têm valor estético e funcional relevante. Quanto mais extensas as ressecções, maior o risco de prejuízos funcionais e cosméticos. Diante disso, torna-se árduo o equilíbrio entre ressecção oncologicamente completa e preservação da função e estética da área acometida (BARAZZETTI DO, et al., 2019).

O principal sítio de localização das lesões de cânceres de pele não melanoma foi a face $(78,1 \%)$, seguida do tronco (13\%). Na face, a predominância no nariz $(29,8 \%)$ e região malar $(21,1 \%)$ corrobora com a literatura. A região conhecida como $\mathrm{H}$, incluindo a face central, região periocular, pálpebra, sobrancelha, 
nariz, perioral, lábio (cutâneo e vermelhão), queixo, mandíbula, orelha, pré-auricular, pós-auricular e pele da têmpora da face, é mais suceptível à ocorrência de carcinomas de pele (ROSSATO LA, et al., 2016). Proporcionalmente, a região facial com maior índice de ressecção com margem cirúrgica comprometida foi a pálpebra, com $46,5 \%$ das ressecções incompletas. Das ressecções em orelha e nariz, $40,9 \%$ e $35,2 \%$, respectivamente, tiveram margem cirúrgica comprometida. A incidência de margens comprometidas na pálpebra pode chegar a 39\%, tendo atingido prevalência superior no presente estudo.

Predominaram lesões do tipo carcinoma basocelular, especialmente do subtipo nodular, porém, proporcionalmente, os carcinomas espinocelulares tiveram maior frequência de margens cirúrgicas incompletas (35\% versus $26,4 \%$ ). Estudo transversal realizado na Austrália, com amostra de 105 pacientes com lesões suspeitas para câncer de pele não melanoma, encontrou prevalências dos tipos histológicos com a seguinte distribuição: $70,2 \%$ das lesões eram carcinoma basocelular, das quais $6 \%$ foram ressecadas com margem cirúrgica comprometida. Não houve ressecção incompleta nas lesões do tipo carcinoma espinocelular (NIGHTINGALE J, et al., 2020). O CBC nodular apresenta alta propensão à ulceração, bem como pior prognóstico, sendo uma variante responsável por $50-79 \%$ dos casos, sendo ainda maior no presente estudo (BARAZZETTI DO, et al., 2019).

Estudo desenvolvido na Itália verificou uma distribuição anatômica dos CBC em homens com predominância em cabeça e pescoço $(79,6 \%)$, tronco $(13,4 \%)$, membros superiores $(3,8 \%)$, membros inferiores $(1,5 \%)$ e genitália $(0,1 \%)$. Nas mulheres, a distribuição foi cabeça e pescoço $(83,9 \%)$, tronco $(9,4 \%)$, membros superiores $(2,5 \%)$, membros inferiores $(2,5 \%)$ e órgãos genitais $(0,2 \%)$ (17). Embora existam muitas variantes clínicas de CBC, os nodulares pigmentados são mais comuns em indivíduos de pele escura e olhos castanhos do que em indivíduos de pele clara e olhos azuis. O CBC superficial geralmente se apresenta nos ombros, costas ou tórax, e múltiplas lesões podem estar presentes ao mesmo tempo. Os subtipos morfeia, micronodular e basoescamoso (também conhecido como metatípico ou misto), são variantes histológicas agressivas e têm alto risco de recorrência (CIVES M, et al., 2020; PASQUALI P, et al., 2020).

Neste estudo, foi verificado também que carcinomas basocelulares dos subtitpos esclerodermiforme e infiltrativo foram significativamente relacionados à ressecção primária incompleta do câncer de pele não melanoma. O subtipo esclerodermiforme de CBC é considerado uma variante histológica agressiva e tem alto risco de recorrência. O subtipo infiltrativo é uma variante semelhante à morfeforma do CBC e é principalmente caracterizada por uma fibrose estromal pesada, com feixes de colágeno densos, que cresce de forma mal circunscrita e frequentemente invade o subcutâneo, enquanto as células tumorais se espalham formando um grande nódulo irregular (CIVES M, et al., 2020).

Da totalidade das lesões identificadas neste estudo, 20\% eram CEC, com predominância do grau I de Broders, seguido do grau II. Apesar de menos prevalentes, os CEC tiveram maior incidência de margem cirúrgica comprometida, sobretudo do subtipo grau II, sendo relevante a associação de lesões do tipo CEC e subtipo Grau II com ressecção primária incompleta. Estudo transversal retrospectivo, desenvolvido em um serviço de Cirurgia Plástica com 145 pacientes com diagnóstico de câncer de pele, identificou uma predominância do grau I de Broders. Em uma análise mais detalhada, dos 38 casos de CEC, 42,1\% pertenciam ao grau I de Broders, $34,21 \%$ ao grau II. Os CECs têm comportamento oncológico mais agressivo, apresentando maior infiltração local e risco e metástase à distância (BROETTO J, et al., 2012).

\section{CONCLUSÃO}

Após análise das exéreses cirúrgicas dos carcinomas de pele incluídos nesse estudo, observou que a índice de margem cirúrgica incompleta foi de $29.8 \%$ e que a exéreses incompletas são significativamente associadas a lesões maiores que $2 \mathrm{~cm}$, localizadas em face e dos subtipos histológicos esclerodermiforme e infiltratava, quando se trata de carcinoma basocelular, e ao subtipo de Broders grau II para carcinoma espinocelular. Espera-se que o presente estudo possar auxiliar o cirurgião na definição das margens cirúrgicas de exéreses de tumores de pele, a fim de evitar ressecção incompleta sem comprometimento de tecido saudável. 


\section{REFERÊNCIAS}

1. BADASH I, et al. Nonmelanoma facial skin cancer: a review of diagnostic strategies, surgical treatment, and reconstructive techniques. Clinical Medicine Insights: Ear, Nose and Throat, 2019; 12: 1179550619865278.

2. BARAZZETTI DO, et al. Quality of life and clinical and demographic characteristics of patients with cutaneous squamous cell carcinoma submitted to tumor resection by double-bladed scalpel. An Bras Dermatol. 2019; 94(3): 304-12.

3. BILDEN TT, et al. Intraoperative Frozen Section Analysis for the Excision of Nonmelanoma Skin Cancer: A SingleCenter Experience. Am Surg. 2019; 85(12): 1397-401.

4. BROETTO J, et al. Tratamento cirúrgico dos carcinomas basocelular e espinocelular: experiência dos Serviços de Cirurgia Plástica do Hospital Ipiranga. Rev Bras Cir Plás. 2012; 27(4): 527-30.

5. CIVES M, et al. Non-Melanoma Skin Cancers: Biological and Clinical Features. Int J Mol Sci. 2020; $21(15)$ : e5394.

6. COLLINS A, et al. Nonsurgical Treatments for Nonmelanoma Skin Cancer. Dermatol Clin. 2019; 37(4): 435-41.

7. DOMÍNGUEZ C, CELIS J. Carcinoma basocelular. Un reto actual para el dermatólogo. Revista Médica Electrónica, 2018; 40(1): 172-182.

8. DUARTE F, et al. Risk factors for development of new skin neoplasms in patients with past history of skin cancer: a survival analysis. Scientific reports, 2018; 8(1): 1-6.

9. FAHRADYAN A, et al. Updates on the Management of Non-Melanoma Skin Cancer (NMSC). Healthcare (Basel). 2017; 5(4): 82-106.

10. GAMONAL AC, et al. Câncer de pele: Prevalência e epidemiologia em um hospital de ensino da cidade de Juiz de Fora-MG. Brazilian Journal of health Review, 2020; 3(6): 15766-15773.

11. HUANG T, et al. Risk factors and outcomes of nonmelanoma skin cancer in children and young adults. The Journal of pediatrics, 2019; 211: 152-158.

12. KALLINI JR, et al. Squamous cell carcinoma of the skin: epidemiology, classification, management, and novel trends. Int J Dermatol. 2015; 54(2): 130-40.

13. LAI V, et al. Epidemiology of skin cancer in the mature patient. Clinics in dermatology, 2018; 36(2): 167-176.

14. MELO R, et al. Ressecção de carcinoma basocelular do tipo metatípico com reconstrução a base de autoenxertia. Brazilian Journal of Development, 2020; 6(12): 95712-95723.

15. NAPOLI J, et al. Estudo epidemiológico da associação entre fatores de risco e excisões incompletas no câncer de pele. Rev. bras. cir. plást, 2021: 40-45.

16. NAHHAS AF, et al. A Review of the Global Guidelines on Surgical Margins for Nonmelanoma Skin Cancers. J Clin Aesthet Dermatol. 2017; 10(4): 37-46.

17. NIGHTINGALE J, et al. Outpatient surgical management of non-melanoma skin cancers of the head and neck in a regional centre: an analysis of costs and outcomes. ANZ J Surg. 2020. Epub ahead of print.

18. PASQUALI P, et al. Use of Cytology in the Diagnosis of Basal Cell Carcinoma Subtypes. J Clin Med. 2020; 9(3): 6128.

19. QUAZI SJ, et al., Surgical Margin of Excision in Basal Cell Carcinoma: A Systematic Review of Literature. Cureus. 2020; 12(7): e9211.

20. ROSSATO LA, et al. Diagnosis of aggressive subtypes of eyelid basal cell carcinoma by 2-mm punch biopsy: prospective and comparative study. Rev Col Bras Cir. 2016; 43(4): 262-9.

21. SILVA P, et al., Tratamento de múltiplos cbc's recidivantes: Importância do rastreio precoce e na qualidade de vida do paciente. Research, Society and Development, 2021; 10(6): e43410615885-e43410615885.

22. SILVA R, DIAS M. Incidência do carcinoma basocelular e espinocelular em usuários atendidos em um hospital de câncer. Revista Família, Ciclos de Vida e Saúde no Contexto Social, 2017; 5(2): 228-234. 